\title{
Anesthesia For an Intoxicated Parturient With a Cervical Collar
}

Jaw Donkoh, Ihuma Ofoma, Ned Nasr, Zerin Dadabhoy, Bozana Alexander

Department of Anesthesiology and Pain Management, J. H. Stroger Hospital of Cook County, Chicago, Illinois 6012, USA

\section{Correspondence}

Bozana Alexander, MD

Anesthesia Research, Room 520

Hektoen

Chicago, IL 60612, USA

Phone: (312) 8644632
Substance abuse has crossed social, economic and geographic borders and presents significant problem that is facing society today. The prevalence of cocaine abuse in young adults (including women) is markedly increased over the past two decades. Approximately ninety percent of cocaine-abusing women are of childbearing age. Thus, it is not surprising to find pregnant women who abuse this drug. During the early months of pregnancy, cocaine abuse may increase the risk of miscarriage. When the drug is used late in pregnancy, it triggers premature labor and may cause an unborn baby to die or to have a stroke, which can result in irreversible brain damage. ${ }^{1}$ Thus, prenatal cocaine exposure may affect infant development. ${ }^{2}$

The diverse clinical manifestations of cocaine abuse combined with physiologic changes of pregnancy, and pathophysiology of coexisting pregnancy-related disease might lead to life-threatening complications and significantly impact the practice of obstetric anesthesia. A complete understanding of the physiology of pregnancy, pathophysiology of pregnancy-specific disorders and anesthetic implications of cocaine abuse in pregnancy is essential for safe anesthetic plan for this high-risk group of patients. ${ }^{3}$

A third-term pregnant woman involved in a traumatic event presents several unique challenges for anesthetic management. We present a-37-week pregnant substance abuser who went into labor after sustaining blunt trauma to the chest. In this instance a decision to provide general anesthesia was complicated by several factors, including a history of rheumatoid arthritis, further compounded by a c-spine that could not be cleared due to her intoxicated condition. The alternative option of regional anesthesia was not practical in a setting of recent traumatic event and inebriated state.

\section{Case Presentation}

A-38-year-old G6P5 parturient presented to the emergency department after involvement in a vehicle collision as a restrained passenger, with chest injury due to air bag deployment. She had a history of rheumatoid arthritis managed with high daily dose of steroids. Initial work up for chest and abdomen was negative. There was concern about a widened mediastinum on chest $\mathrm{x}$-ray, which was cleared by the radiologist after CT scan. C-spine instability could not be excluded and a cervical collar was placed by the trauma service. A toxicology report was positive for heroine and cocaine. The Anesthesiology on call team was consulted shortly after the patient's arrival to the labor and delivery unit. We were informed that the patient was demonstrating changes in mental status including periodic drowsiness.

We found distressed female in labor pain demanding a csection. She revealed that she had general anesthesia once for a cesarean section. She denied food ingestion in the last five hours. On inspection the airway appeared manageable despite the presence of a cervical collar, which necessitated meticulous planning of airway management. After discussing the situation with the obstetrical team a decision was made to allow labor to progress, with surgical intervention if necessary. Aspiration prophylaxis was administered accordingly. Availability of an emergency airway cart in the operating room was verified. The anesthesia team was summoned again three hours later for emergency cesarean section due to failure of labor to progress. Patient was placed supine in the LUD position with a roll under her shoulders and pre-oxygenated with her head at a 20-degree elevation. She underwent uneventful rapid sequence induction and was subsequently intubated with c-collar in place and in line neck stabilization. A stress dose of steroids was administered. Anesthesia was maintained with sevoflurane in nitrous oxide and oxygen. 
A male infant was delivered with APGAR scores 8 and 9. At the end of the procedure patient met criteria for extubation, which was uneventful, as was the immediate recovery period.

\section{Discussion}

When selecting an anesthesia technique each case must be analyzed individually and particular issues addressed accordingly. ${ }^{3}$ For instance, the airway tends to be more edematous and vascular during pregnancy, and rheumatoid arthritis may affect synovial joints in the c-spine. Even in the absence of a c-collar, avoidance of neck extension would still be prudent and a smaller size tube recommended. Cocaine abuse is known to have effects on hemodynamic status and possibly hemostasis. ${ }^{4}$ In our intoxicated preg- nant trauma patient, we chose balanced general anesthesia, which led to a favorable outcome for both the mother and the fetus.

\section{References}

1. Slutsker L. Risks associated with cocaine use during pregnancy. Obstet Gynecol 1992;778-89.

2. Richardson GA, Goldschmidt L, Willford J. The effects of prenatal cocaine use on infant development. Neurotoxicol Teratol 2008;30:96-106.

3. Kuczkowski KM. Cocaine abuse in pregnancy-anesthetic implications. Int J Obstet Anesth 2002;11:204-10.

4. Chang A. Trauma in pregnancy. Emerg Med Clin N Amer 2009;16:209-28. 